\title{
Effect of Pair Breaking on Mesoscopic Persistent Currents Well above the Superconducting Transition Temperature
}

\author{
H. Bary-Soroker, ${ }^{1 *}$ O. Entin-Wohlman, ${ }^{2,3}$ and Y. Imry ${ }^{1}$ \\ ${ }^{1}$ Department of Condensed Matter Physics, Weizmann Institute of Science, Rehovot 76100, Israel \\ ${ }^{2}$ Department of Physics, Ben Gurion University, Beer Sheva 84105, Israel \\ ${ }^{3}$ Albert Einstein Minerva Center for Theoretical Physics, Weizmann Institute of Science, Rehovot 76100, Israel
}

(Received 2 April 2008; published 28 July 2008)

\begin{abstract}
We consider the mesoscopic normal persistent current (PC) in a very low-temperature superconductor with a bare transition temperature $T_{c}^{0}$ much smaller than the Thouless energy $E_{c}$. We show that in a rather broad range of pair-breaking strength, $T_{c}^{0} \lesssim \hbar / \tau_{s} \lesssim E_{c}$, the transition temperature is renormalized to zero, but the PC is hardly affected. This may provide an explanation for the magnitude of the average PC's in the noble metals, as well as a way to determine their $T_{c}^{0}$ 's.
\end{abstract}

Introduction. - The magnitude of the equilibrium averaged persistent currents (PC's) $[1,2]$ in normal metals has been a long-standing puzzle. Experiments [3-5] produce a current larger by at least 2 orders of magnitude than the theoretical prediction for noninteracting electrons [6-8] and seem to indicate that the low-flux response is diamagnetic. The average PC of a diffusive system with interactions was calculated first in this connection [9] in Refs. [10,11]. The resulting PC was found to be much larger than that of a noninteracting system, but nevertheless not large enough to explain the experiments.

Repulsive electron-electron interactions [11] result in a paramagnetic response (at small magnetic fluxes) whose magnitude is smaller than the experiment by about a factor of 5. This disagreement is due to the downward renormalization of the interaction [12,13]. Attractive interactions [10] result in a diamagnetic response, whose magnitude (due to the very low superconducting transition temperature), is again smaller by a factor of order five than the measured one. This is in spite of the renormalization upward of the attractive interaction. Attractive interactions, at low energies, imply (with no pair breaking) a transition into a superconducting state, and the PC of such an interacting system depends on its transition temperature. These temperatures are very low [14] for the noble metals used in the PC experiments - hence the too small predicted values for the PC.

Here we consider attractive interactions. We show that the presence of a very small amount of pair breakers, e.g., magnetic impurities (which seem to be very difficult to avoid in these metals [15]), may change the picture profoundly. Obviously, one may consider other pair breakers, such as a two-level systems [16] or simply a magnetic field [17]. In this Letter we treat specifically the case of magnetic impurities. We find that within a significant range of the pair-breaking strength, the magnetic impurities suppress the transition temperature down to immeasurable values, leaving concomitantly the PC almost unchanged.
The physical reason for this remarkable observation is that the PC is determined by the interaction on the scale of the Thouless energy $E_{c}=\hbar D / L^{2}(\sim 20 \mathrm{mK}$ for a typical experimental system), while the bare transition temperature, $T_{c}^{0}$, is much smaller. (The circumference of the ring is denoted by $L$ and $D$ is the diffusion coefficient.) This gives rise to a rather wide range of pair-breaking strengths, presented here by the spin-scattering time $\tau_{s}$,

$$
T_{c}^{0} \lesssim \hbar / \tau_{s} \lesssim E_{c},
$$

in which the actual transition temperature $T_{c}$ will drop to zero [18], but the PC will be hardly affected. As a result, it is the bare transition temperature of the system without the magnetic impurities, $T_{c}^{0}$, as opposed to $T_{c}$, which dominates the expression for PC, see Fig. 1. We conclude here [19] on the experimental results of Ref. [3]. In order to explain them, it is necessary to assume a $T_{c}^{0}$ in the $1 \mathrm{mK}$ range for copper. Our basic assertion is that this may indeed be the correct order of magnitude of $T_{c}^{0}$ for ideally clean copper, but that it is knocked down to zero or to a very low value by a minute, $\lesssim$ ppm, amount of unwanted [15] pair breakers. We emphasize, however, that our result concerning the fundamentally different sensitivities of $T_{c}$ and $\mathrm{PC}$ to pair breaking in the range given by Eq. (1),

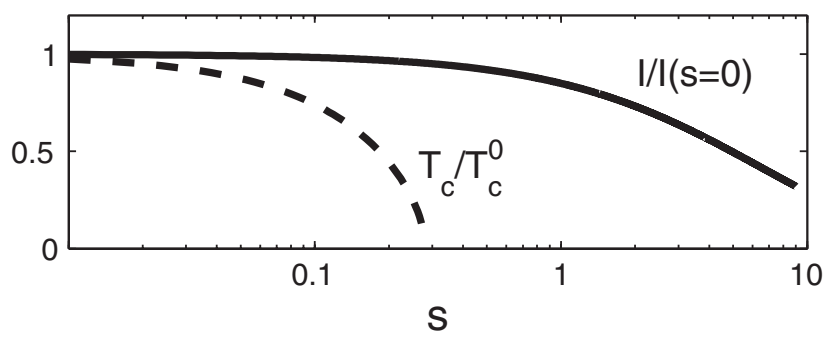

FIG. 1. The first flux harmonic [ $m=1$; see Eq. (2)], in units of $I(s=0)$, of the PC at $T=E_{c}$ (full line) and $T_{c} / T_{c}^{0}$ (dashed line) as functions of the pair-breaking strength, $s=1 /\left(\pi T_{c}^{0} \tau_{s}\right)$, displayed on a logarithmic scale. 
remains valid regardless of the situation in specific materials. The Kondo screening of the spins is not considered here. Other effects of magnetic impurities have previously been considered in Ref. [20].

Results. - The expression we obtained for the PC in a diffusive ring with magnetic impurities can be expressed as a sum over the harmonics of the magnetic flux through the ring $\phi$, in units of the flux quantum $h / e$,

$$
\begin{aligned}
I= & -8 e E_{c} \sum_{m=1}^{\infty} \frac{\sin (4 \pi m \phi)}{m^{2}} \\
& \times \sum_{\nu} \int_{0}^{\infty} d x \frac{x \sin (2 \pi x) \Psi^{\prime}(F(x, \nu))}{\ln \left(T / T_{c}^{0}\right)+\Psi(F(x, \nu))-\Psi\left(\frac{1}{2}\right)}, \\
F(x, \nu)= & \frac{1}{2}+\frac{|\nu|+2 / \tau_{s}}{4 \pi T}+\frac{\pi E_{c} x^{2}}{m^{2} T},
\end{aligned}
$$

(using $\hbar=1$ ). Here $\nu$ denotes the bosonic Matsubara frequency [21], $\Psi$ and $\Psi^{\prime}$ are the digamma function and its derivative, and $T$ is the temperature. Our expression (2) generalizes the result of Ref. [10] for the case where spinscattering is present: the Matsubara frequency $|\nu|$ is shifted by $2 / \tau_{s}$. However, the superconducting transition temperature (which appears formally in the denominator of the integrand) is not the one modified by the pair breakers, but retains its bare (magnetic impurities free) value. Interestingly enough, it follows that by measuring the PC one may determine $T_{c}^{0}$ (which would be directly measurable only if all low-temperature pair breaking could be eliminated).

In Fig. 2 the PC is plotted using Eq. (2). At the critical pair-breaking time $1 / \tau_{s} \simeq T_{c}^{0}$, corresponding to $s=$ $1 / \pi \tau_{s} T_{c}^{0} \simeq 1 / \pi$, the transition temperature vanishes [18], while the PC is hardly affected. The measured PC in the copper samples of Ref. [3] is $I\left(T \lesssim E_{c}\right) \simeq-e E_{c}$. The curve with $s=1$ in the upper panel, taken with $T_{c}^{0}=$ $1.5 \mathrm{mK}$ and $E_{c}=15 \mathrm{mK}$ (the value for the samples of Ref. [3]) gives a PC lower by only $25 \%$. A better fit is possible by changing the parameters somewhat, but we do not regard this as crucial at the present stage. Likewise, we can qualitatively explain the result of Ref. [4]. The high frequency results of Ref. [5] require a separate discussion [5]. The PC is reduced significantly once $1 / \tau_{s} \geq E_{c}$, or $L_{s} \equiv \sqrt{D \tau_{s}} \leq L$. For $T_{c}^{0} / E_{c}=0.1(0.01)$, the condition for $E_{c} \tau_{s} \sim 1$ is $s=10(100)$.

Derivation.-For completeness, we outline below the derivation of the $\mathrm{PC}$ in the presence of magnetic scattering [19]. The PC, Eq. (2), is obtained by differentiating the free energy with respect to the flux. Our system is described by the Hamiltonian [18]

$$
\begin{aligned}
\mathcal{H}= & \int d \mathbf{r}\left(\psi_{\alpha}^{\dagger}(\mathbf{r})\left[\left(\mathcal{H}_{0}+u_{1}(\mathbf{r})\right) \delta_{\alpha \gamma}+u_{2}(\mathbf{r}) \mathbf{S} \cdot \boldsymbol{\sigma}^{\alpha \gamma}\right] \psi_{\gamma}(\mathbf{r})\right. \\
& \left.-\frac{g}{2} \psi_{\alpha}^{\dagger}(\mathbf{r}) \psi_{\gamma}^{\dagger}(\mathbf{r}) \psi_{\gamma}(\mathbf{r}) \psi_{\alpha}(\mathbf{r})\right),
\end{aligned}
$$

in which the last term is the attractive interaction, of
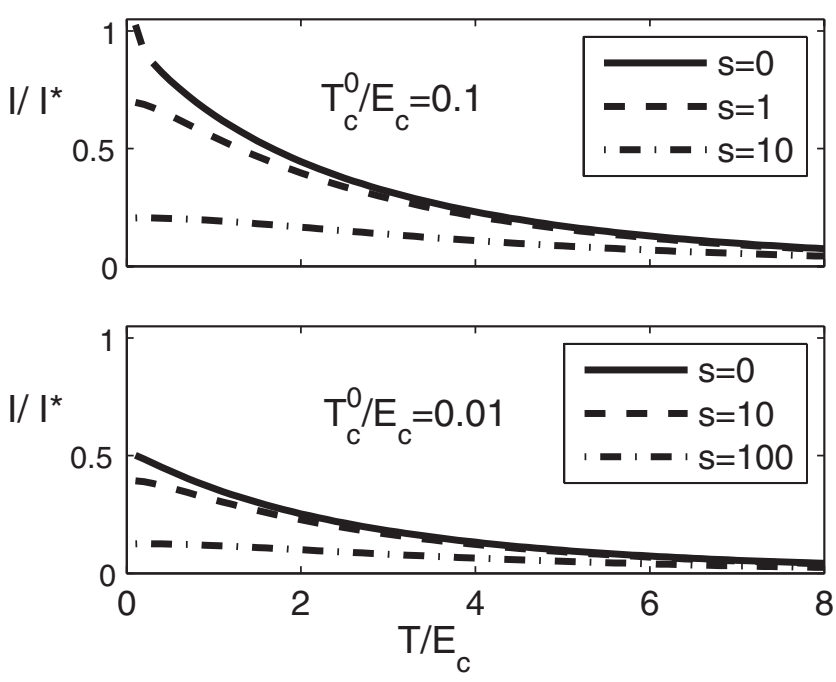

FIG. 2. The first flux harmonic of the PC in units of $I^{*}=$ $-e E_{c}$ as a function of the temperature, for two values of $T_{c}^{0} / E_{c}$ and several values of $s$. Keeping, at $T \lesssim E_{c}$, up to the 100 lowest values of $|\nu|$, was necessary for convergence. Note that the $s=0$ curve in the upper panel is valid only for $T / T_{c} \geq 1+\mathrm{Gi}$, where $\mathrm{Gi}$ is the Ginzburg parameter $(\mathrm{Gi} \sim 0.1$ for the samples of Ref. [3]).

coupling $g$. The spin components are $\alpha$ and $\gamma, \boldsymbol{\sigma}$ is the vector of the Pauli matrices, and $\mathcal{H}_{0}=(-i \boldsymbol{\nabla}-$ $e \mathbf{A})^{2} / 2 m-\mu$ ( $\mu$ is the chemical potential and $\mathbf{A}$ is the vector potential describing the flux through the ring). The scattering, both nonmagnetic and magnetic, is assumed to result from $N_{i}$ pointlike impurities, such that

$$
u_{1}(\mathbf{r})+u_{2}(\mathbf{r}) \mathbf{S} \cdot \boldsymbol{\sigma} \equiv \sum_{i=1}^{N_{i}}\left(\delta\left(\mathbf{r}-\mathbf{R}_{i}\right)-\frac{1}{V}\right)\left(u_{1}+u_{2} \mathbf{S}_{\mathbf{R}_{i}} \cdot \boldsymbol{\sigma}\right),
$$

where $V$ is the system volume. In averaging over the impurity disorder one assumes that the impurity locations, $\mathbf{R}_{i}$, are random, and so are their classical spins, such that $\left\langle\mathbf{S}_{\mathbf{R}_{i}}\right\rangle=0$, and $\left\langle\mathbf{S}_{\mathbf{R}_{i}} \cdot \mathbf{S}_{\mathbf{R}_{j}}\right\rangle=\delta_{i j} S(S+1)$.

The partition function, $Z$, is calculated by the method of Feynman path integrals [22], combined with the Grassman algebra of many-body fermionic coherent states in terms of the variables $\psi_{\alpha}(\mathbf{r}, \tau)\left(\bar{\psi}_{\alpha}(\mathbf{r}, \tau)\right)$. Introducing the bosonic fields $\Delta(\mathbf{r}, \tau)$ via the Hubbard-Stratonovich transformation leads to the partition function $Z=\int D(\psi(\mathbf{r}, \tau)$, $\bar{\psi}(\mathbf{r}, \tau)) D\left(\Delta(\mathbf{r}, \tau), \Delta^{*}(\mathbf{r}, \tau)\right) e^{-\mathcal{S}}$ with

$$
\mathcal{S}=\int d \mathbf{r} \int_{0}^{\beta} d \tau\left(\frac{|\Delta(\mathbf{r}, \tau)|^{2}}{g}-\frac{1}{2} \bar{\Psi}(\mathbf{r}, \tau) G_{\mathbf{r}, \mathbf{r} ; \tau, \tau}^{-1} \Psi(\mathbf{r}, \tau)\right),
$$

where $\bar{\Psi}=\left(\bar{\psi}_{\uparrow}, \bar{\psi}_{\downarrow}, \psi_{\uparrow}, \psi_{\downarrow}\right)$. The inverse Green function $G^{-1}$ (at equal positions $\mathbf{r}$ and equal imaginary times $\tau$ ) is given by 


$$
G_{\mathbf{r}=\mathbf{r}^{\prime} ; \tau=\tau^{\prime}}^{-1}=\left[\begin{array}{cccc}
-\partial_{\tau}-h_{\uparrow}^{\phi} & -2 u_{2} S_{-} & 0 & \Delta \\
-2 u_{2} S_{+} & -\partial_{\tau}-h_{\downarrow}^{\phi} & -\Delta & 0 \\
0 & -\Delta^{*} & -\partial_{\tau}+h_{\uparrow}^{-\phi} & 2 u_{2} S_{+} \\
\Delta^{*} & 0 & 2 u_{2} S_{-} & -\partial_{\tau}+h_{\downarrow}^{-\phi}
\end{array}\right] \equiv\left[\begin{array}{cc}
\hat{G}_{p}^{-1} & \hat{\Delta} \\
\hat{\Delta}^{\dagger} & \hat{G}_{h}^{-1}
\end{array}\right],
$$

where $h_{\alpha}^{ \pm \phi}=\mathcal{H}_{0}( \pm \mathbf{A})+u_{1}+\operatorname{sgn}(\alpha) S_{z} u_{2}$, and $S_{ \pm}=\left(S_{x} \pm i S_{y}\right) / 2$.

The integration over the fermionic part of the action (5) yields

$$
Z=\int D\left(\Delta(\mathbf{r}, \tau), \Delta^{*}(\mathbf{r}, \tau)\right) \exp \left(\frac{1}{2} \operatorname{Tr} \ln \left(\beta G^{-1}\right)-\int d \mathbf{r} \int_{0}^{\beta} d \tau \frac{|\Delta(\mathbf{r}, \tau)|^{2}}{g}\right)
$$

In order to treat the boson fields $\Delta$, we expand $\operatorname{Tr} \ln \left(\beta G^{-1}\right)$ up to second order in $\Delta$. This expansion is valid for temperatures well above the transition temperature, and, strictly speaking, above the Ginzburg critical region. The zeroth order is omitted as it leads to the tiny magnitude PC of noninteracting, grand-canonical, normal metal rings [6]. The result in Fourier space reads (the dependence on the magnetic flux is specified below)

$$
\left.\operatorname{Tr} \ln \left(\beta G^{-1}\right)\right|^{2 \mathrm{nd}}=-\sum_{\mathbf{q}_{1}, \mathbf{q}_{2}, \nu} \sum_{\mathbf{k}_{1}, \mathbf{k}_{2}, \omega} \operatorname{Tr}\left[\hat{G}_{p}\left(\mathbf{k}_{1}+\mathbf{q}_{1}, \mathbf{k}_{2}+\mathbf{q}_{2}, \omega+\nu\right) \hat{\Delta}\left(\mathbf{q}_{2}, \nu\right) \hat{G}_{h}\left(\mathbf{k}_{2}, \mathbf{k}_{1},-\omega\right) \hat{\Delta}^{\dagger}\left(\mathbf{q}_{1}, \nu\right)\right] .
$$

The resulting expression for the partition function may be simplified considerably. First, the terms that survive the disorder-average in the sum of Eq. (8) are those for which [23] $\mathbf{q}_{1}=\mathbf{q}_{2}$. Second, the particle and the hole Green functions, $\hat{G}_{p}$ and $\hat{G}_{h}$, [see Eq. (6)] are related,

$$
\hat{G}_{h}\left(\mathbf{k}, \mathbf{k}^{\prime} ; \omega\right)=-\hat{G}_{p}^{t}\left(-\mathbf{k},-\mathbf{k}^{\prime}, \omega\right),
$$

where the superscript $t$ denotes the transposed. Carrying out the integration in Eq. (7),

$$
Z=\prod_{\mathbf{q}, \nu} \mathcal{N}(0)\left(\frac{V}{g}-T \Pi(\mathbf{q}, \nu)\right)^{-1},
$$

where $\mathcal{N}(0)$ denotes the extensive density of states at the Fermi level. The polarization is

$$
\Pi(\mathbf{q}, \nu)=\frac{1}{2} \sum_{\omega} \varepsilon_{\alpha \gamma} K_{\omega \alpha \gamma}(\mathbf{q}, \nu)
$$

with

$$
\begin{aligned}
K_{\omega \alpha \gamma}(\mathbf{q}, \nu)= & \sum_{\mathbf{k}_{1}, \mathbf{k}_{2}}\left\langle G_{\alpha \alpha^{\prime}}\left(\mathbf{k}_{1}+\mathbf{q}, \mathbf{k}_{2}+\mathbf{q}, \omega+\nu\right)\right. \\
& \left.\times \varepsilon_{\alpha^{\prime} \gamma^{\prime}} G_{\gamma \gamma^{\prime}}\left(-\mathbf{k}_{2},-\mathbf{k}_{1},-\omega\right)\right\rangle .
\end{aligned}
$$

Here $\varepsilon$ is the antisymmetric tensor, $\varepsilon_{\alpha \alpha}=0$, and $\varepsilon_{\uparrow \downarrow}=$ $-\varepsilon_{\Downarrow}=1$, and $G$ denotes the particle Green function.

In Ref. [18] $K(0,0)$ was calculated using a Dyson equation. We generalize their calculation to obtain $K(\mathbf{q}, \nu)$ and, consequently, the polarization becomes [19]

$$
\begin{aligned}
\frac{T}{\mathcal{N}(0)} \Pi(\mathbf{q}, \nu)= & \Psi\left(\frac{1}{2}+\frac{\omega_{D}}{2 \pi T}+\frac{|\nu|+D \mathbf{q}^{2}}{4 \pi T}\right) \\
& -\Psi\left(\frac{1}{2}+\frac{D \mathbf{q}^{2}+|\nu|+2 / \tau_{s}}{4 \pi T}\right) .
\end{aligned}
$$

Here $\omega_{D}$ is the cutoff frequency on the attractive interaction, and the pair-breaking time $\tau_{s}$ is given by

$$
\frac{1}{\tau_{s}}=2 \pi \mathcal{N}(0) N_{i} S(S+1) u_{2}^{2} .
$$

The transition temperature of the system in the absence of pair breakers, $T_{c}^{0}$, is obtained from the $\mathbf{q}=0, \nu=0$ pole of $Z$, upon setting $1 / \tau_{s}=0$,

$$
\frac{V}{g \mathcal{N}(0)}=\Psi\left(\frac{1}{2}+\frac{\omega_{D}}{2 \pi T_{c}^{0}}\right)-\Psi\left(\frac{1}{2}\right)
$$

(Note that the same procedure in the presence of the pair breaking reproduces the decrease in the transition temperature $T_{c}$, as found in Ref. [18].) Since $\omega_{D} \gg T_{c}^{0}, T$ we may use the asymptotic expansion of the digamma function. In this way we obtain

$$
Z=\prod_{\mathbf{q}, \nu}\left[\ln \left(\frac{T}{T_{c}^{0}}\right)+\Psi\left(\frac{1}{2}+\frac{D \mathbf{q}^{2}+|\nu|+2 / \tau_{s}}{4 \pi T}\right)-\Psi\left(\frac{1}{2}\right)\right]^{-1} .
$$

Finally, the PC is given by $I=(e / h) \partial T \ln Z / \partial \phi$. In our ring geometry, the flux enters the longitudinal component, $q_{\|}$, of the vector $\mathbf{q}$ as

$$
q_{\|}=\frac{2 \pi}{L}(n+2 \phi)
$$

where $n$ is an integer. Only the zero transverse momentum contributes significantly to the current. Our result (2) is obtained upon inserting Eq. (16) into the definition of the current and employing the Poisson summation formula. It then follows from Eq. (2) that values of $\tau_{s}$ which are detrimental to $T_{c}$, may hardly affect the PC (see Fig. 1).

We conclude by further explaining the physical argument behind our result. Very roughly, the renormalization of the dimensionless attractive interaction $\lambda(>0)$ from a higher frequency scale $\omega_{>}$to a lower one, $\omega_{<}$, is given by $\lambda\left(\omega_{<}\right)=\frac{\lambda\left(\omega_{>}\right)}{1-\lambda\left(\omega_{>}\right) \ln \left(\frac{\omega_{>}}{\omega_{<}}\right)}$. At $T_{c}^{0}$ and $1 / \tau_{s}=0$, the attractive 
interaction should diverge. Using this to eliminate $\lambda\left(\omega_{D}\right)$ $(\equiv g N(0) / V)$, we obtain that for $T_{c}^{0} \lesssim \omega \ll \omega_{D}, \lambda(\omega) \backsim$ $1 / \ln \left(\omega / T_{c}^{0}\right)$, which around the Thouless scale, is close to the value found in Ref. [10]. The pair breaking stops the renormalization at $1 / \tau_{s}$, but does not significantly change the interaction on the much larger scale of $E_{c}$. Our prediction can also be tested with very small rings made of known low $T_{c}$ superconductors.

We point out that the mechanism suggested by Kravtsov and Altshuler [24], relating extrinsic dephasing to an enhanced PC, is different than ours, since it relies on the rectification of the noise.

We thank E. Altman, A. M. Finkel'stein, L. Gunther, K. Michaeli, A.C. Mota, F. von Oppen, Y. Oreg, G. Schwiete, and A. A. Varlamov for very helpful discussions. This work was supported by the German Federal Ministry of Education and Research (BMBF) within the framework of the German-Israeli project cooperation (DIP), and by the Israel Science Foundation (ISF).

*hamutal.soroker@weizmann.ac.il

[1] M. Büttiker, Y. Imry, and R. Landauer, Phys. Lett. A 96, 365 (1983).

[2] Y. Imry, Introduction to Mesoscopic Physics (Oxford University Press, Oxford, 2002), 2nd ed.

[3] L. P. Levy, G. Dolan, J. Dunsmuir, and H. Bouchiat, Phys. Rev. Lett. 64, 2074 (1990).

[4] E. M. Q. Jariwala, P. Mohanty, M. B. Ketchen, and R. A. Webb, Phys. Rev. Lett. 86, 1594 (2001).

[5] R. Deblock, R. Bel, B. Reulet, H. Bouchiat, and D. Mailly, Phys. Rev. Lett. 89, 206803 (2002).

[6] H. F. Cheung, E. K. Riedel, and Y. Gefen, Phys. Rev. Lett. 62, 587 (1989).

[7] E. K. Riedel and F. von Oppen, Phys. Rev. B 47, 15449 (1993).

[8] B. L. Altshuler, Y. Gefen, and Y. Imry, Phys. Rev. Lett. 66, 88 (1991).

[9] The fluctuation correction to the orbital magnetic response above $T_{c}$ was calculated first by L. G. Aslamazov and A. I. Larkin, Sov. Phys. JETP 40, 321 (1974).
[10] V. Ambegaokar and U. Eckern, Europhys. Lett. 13, 733 (1990).

[11] V. Ambegaokar and U. Eckern, Phys. Rev. Lett. 65, 381 (1990).

[12] P. G. de Gennes, Superconductivity of Metals and Alloys (Addison-Wesley Publishing Co., Reading, MA, 1989).

[13] P. Morel and P. W. Anderson, Phys. Rev. 125, 1263 (1962).

[14] The $T_{c}^{\prime} s$ of the noble metals were estimated using varying amounts of alloying by R.F. Hoyt and A.C. Mota, Solid State Commun. 18, 139 (1976). The pair-breaking strengths in these alloys are not precisely known.

[15] F. Pierre, A. B. Gougam, A. Anthore, H. Pothier, D. Esteve, and N.O. Birge, Phys. Rev. B 68, 085413 (2003). This work highlighted experimentally the role of minute amounts of magnetic impurities in producing the extra low-temperature dephasing in the noble metal samples.

[16] Y. Imry, H. Fukuyama, and P. Schwab, Europhys. Lett. 47, 608 (1999).

[17] G. Schwiete and Y. Oreg (to be published), have considered the strong Little-Parks effect including fluctuations for rings shorter than the coherence length. There the $T_{c}$ is driven to zero due to the pair-breaking effect of the flux. Like in our case, the PC can still be large outside the superconducting regime. These results are relevant to recent experiments in $\mathrm{Al}$ rings; see N.C. Koshnick, H. Bluhm, M.E. Huber, and K. A. Moler, Science 318, 1440 (2007).

[18] A. A. Abrikosov and L. P. Gorkov, Sov. Phys. JETP 12, 1243 (1961).

[19] More details about the derivation and the results, including comparison with Refs. [4,5], will be given elsewhere.

[20] U. Eckern and P. Schwab, J. Low Temp. Phys. 126, 1291 (2002).

[21] The classical limit, i.e., retaining only the lowest Matsubara frequency $\nu=0$, holds only when the temperature $T$ is larger than $E_{c}$ (see Fig. 1 in Ref. [10]).

[22] A. Altland and B. Simons, Condensed Matter Field Theory (Cambridge University Press, Cambridge, 2006).

[23] A. A. Abrikosov, L. P. Gorkov, and I. E. Dzyaloshinski, Methods of Quantum Field Theory in Statistical Physics (Prentice-Hall, Englewood Cliffs, NJ, 1963).

[24] V.E. Kravtsov and B. L. Altshuler, Phys. Rev. Lett. 84, 3394 (2000). 